Check for updates

Cite this: Phys. Chem. Chem. Phys., 2021, 23, 19571

\section{Designing new ferromagnetic double perovskites: the coexistence of polar distortion and half-metallicity}

\author{
Neda Rahmani, (DD *a Mohammad Ebrahim Ghazi, (D) ${ }^{\mathrm{b}}$ Morteza Izadifard, (D) ${ }^{\mathrm{b}}$ \\ Alireza Shabani iD a and Jost Adam iD *a
}

\begin{abstract}
Advancing technology and growing interdisciplinary fields raise the need for new materials that simultaneously possess several significant physics quantities to meet human demands. In this research, using density functional theory, we aim to design $\mathrm{A}_{2} \mathrm{MnVO}_{6}(\mathrm{~A}=\mathrm{Ca}, \mathrm{Ba})$ as new double perovskites and investigate their structural, electronic, and magnetic properties. Structural calculations based on the total energies show the optimized monoclinic and orthorhombic crystal structures for the $\mathrm{Ca}_{2} \mathrm{MnVO}_{6}(\mathrm{CMVO})$ and $\mathrm{Ba}_{2} \mathrm{MVO}_{6}$ (BMVO) compounds, respectively. Through performing calculations, we reveal that the Jahn-Teller effect plays an important role in polar distortions of $\mathrm{VO}_{6}$ and elongation of $\mathrm{MnO}_{6}$ octahedra, resulting from the $\mathrm{V}^{5+}\left(3 \mathrm{~d}^{0}\right)$ and $\mathrm{Mn}^{3+}\left(3 \mathrm{~d}^{4}: t_{2 g}^{3} \mathrm{e}_{\mathrm{g}}^{1}\right)$ electron configurations. The spin-polarized calculations predict the half-metallic ferromagnetic ground state for CMVO and BMVO with a total magnetic moment of $4.00 \mu_{\mathrm{B}}$ f.u. ${ }^{-1}$ Our findings introduce CMVO and BMVO double perovskites as promising candidates for designing ferromagnetic polar half-metals and spintronic applications.
\end{abstract}

Received 2nd June 2021 Accepted 29th July 2021

DOI: $10.1039 / \mathrm{d} 1 \mathrm{cp} 02479 \mathrm{e}$

rsc.li/pccp ferromagnetic (FM) and colossal magnetoresistance. ${ }^{3-5}$ These materials have been widely used in different technological fields such as magneto-optic device materials, memory devices, and spintronics. ${ }^{6,7}$

The interest in studying double perovskite materials has taken off since the discovery of the $\mathrm{HM}$ property in $\mathrm{Sr}_{2} \mathrm{FeMoO}_{6}$ with room-temperature transition. ${ }^{8}$ The $\mathrm{HM}$ materials are a class of materials that demonstrate a metallic character in one spin channel while behaving as an insulator or semiconductor for the other spin channel. The $100 \%$ spin polarization of these materials at the Fermi level makes them suitable candidates for future high-performance spintronic devices characterized by the transport of spin, or charge and spin. The spin polarization is defined as the net fractional spin polarization near the Fermi level denoted by:

$$
P=\frac{d_{\uparrow}-d_{\downarrow}}{d_{\uparrow}+d_{\downarrow}}
$$

where $d_{\uparrow}$ and $d_{\downarrow}$ represent the density of states (DOS) for spin up and down channels, respectively. ${ }^{9}$

Another material class with potential for developing multifunctional devices, which have been the focus of intensive investigation in the past decades, is multiferroics (MFs). MFs are materials in which several ferroic orders (e.g. magnetic and electric) coexist in a single phase. MFs are considered rare materials due to the incompatibility between the origin of ferromagnetism and ferroelectricity, in which ferromagnetism

\footnotetext{
${ }^{a}$ Department of Mechanical and Electrical Engineering, University of Southern Denmark,DK-640o Sønderborg, Denmark.E-mail: neda@sdu.dk, jostadam@sdu.dk

${ }^{b}$ Faculty of Physics, Shahrood University of Technology, Shahrood, Iran
} 

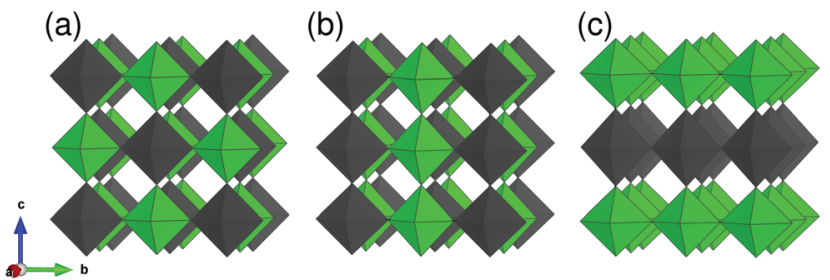

Fig. $1 \mathrm{BO}_{6}$ and $\mathrm{B}^{\prime} \mathrm{O}_{6}$ octahedra ordering schemes in $\mathrm{A}_{2} \mathrm{BB}^{\prime} \mathrm{O}_{6}$ double perovskites: (a) rock-salt, (b) columnar and (c) layered orderings. The $\mathrm{BO}_{6}$ and $\mathrm{B}^{\prime} \mathrm{O}_{6}$ octahedra are represented with two different colors.

typically contains partially filled d orbitals, while ferroelectricity prefers empty $d$ orbitals. One way to overcome the $d^{0}-d^{n}$ dilemma and find new MFs is employing double perovskite structures in which one of the sublattices is occupied with magnetic ions and the other one is occupied with FE ions. ${ }^{10}$

Recently, another mutually exclusive set of properties, polarity/ ferroelectricity and metallicity, has attracted significant attention and several investigations have been made on the polar metals. ${ }^{11-13}$ The exploitation of this material class with contraindicated properties in the same system is intriguing from both technological and fundamental perspectives. To date, very few studies have been done on polar half-metals and MF half-metals, and the discovery of these materials remains challenging. ${ }^{14-16}$

Based on these premises, herein, we attempt to design two new multifunctional double perovskite compounds, $\mathrm{Ca}_{2} \mathrm{MnVO}_{6}$ (CMVO) and $\mathrm{Ba}_{2} \mathrm{MnVO}_{6}$ (BMVO), and provide a theoretical study on their structural, electronic, and magnetic properties using first-principles calculations. The possible structural degrees of freedom, including different crystal structures and various $B$ and $\mathrm{B}^{\prime}$ cationic site arrangements, are investigated to find the optimized ground state with minimum energy. The electronic analysis is supplied based on the Jahn-Teller (JT) effects for interpreting the structural polar distortion of the $\mathrm{B}\left(\mathrm{B}^{\prime}\right) \mathrm{O}_{6}$ octahedral cage. Employing spin-polarized calculations, we predict an HM-FM ground state for two proposed compounds. In the end, we represent the electronic charge density difference to visualize the nature of the bond character and explain the charge transfer in the studied double perovskites. Our investigations predict concurrence of half-metallicity and polar nature in CMVO and BMVO double perovskites, which is a rare behaviour, making them suitable candidates for future multifunctional applications.

\section{Computational methods}

We employed density functional theory (DFT) implemented in the SIESTA code, which is a fully self-consistent method based on a linear combination of atomic orbitals (LCAO) technique with double $-\zeta$ plus polarization (DZP) basis set, for these investigations. ${ }^{17-19}$ To study the structural, electronic and magnetic properties of double perovskites $\mathrm{A}_{2} \mathrm{MnVO}_{6}$ $(\mathrm{A}=\mathrm{Ca}, \mathrm{Ba})$, we employed the generalized gradient approximation (GGA), as formulated by Perdew-Burke-Ernzerhof (PBE) to approximate the exchange-correlation functional. ${ }^{20}$
Structural optimizations were performed for both lattice parameters and atomic positions by employing a conjugate gradient algorithm. We applied the Monkhorst-Pack scheme ${ }^{21}$ with $7 \times$ $7 \times 5 k$-points for sampling the Brillouin zone and a mesh cutoff of 300 Ry for all calculations. The spin-polarized calculations were performed in all cases. The geometries were optimized until the maximum atomic force reached values less than $0.01 \mathrm{eV}^{-1}$ with an energy convergence threshold of $1 \times$ $10^{-4} \mathrm{eV}$. Since this study intends to introduce new double perovskite compounds and investigate their properties, we considered the most used XC functional (GGA-PBE). As a well-known limitation of the DFT method, GGA approximation underestimates the band gaps of solids. ${ }^{22}$ To correct this systematic error and accurately investigate the electronic properties of the proposed compounds, we have taken into account the on-site Coulomb repulsion $(\mathrm{GGA}+U)$ for all calculations due to the presence of highly localized $3 \mathrm{~d}$ orbitals of $\mathrm{Mn}$ and $\mathrm{V}$ transition metals. ${ }^{23}$ To the best of our knowledge, in the case of our new double perovskites $\mathrm{A}_{2} \mathrm{MnVO}_{6}(\mathrm{~A}=\mathrm{Ca}, \mathrm{Ba})$, there is no experimentally measured bandgap in the literature to verify our theoretical results. Thus, we utilized the Hubbard parameter $U$ for strongly correlated $3 \mathrm{~d}$ electrons with the values of $3,4 \mathrm{eV}$ for $\mathrm{V}$ and $4,5 \mathrm{eV}$ for $\mathrm{Mn}$ taken from the similar compounds in the previous studies. ${ }^{24-27}$

\section{Results and discussion}

\subsection{Crystal structure and stability}

To find the ground state crystal structure of CMVO and BMVO double perovskites we considered a tetragonal crystal structure with lattice parameters similar to the $\mathrm{Sr}_{2} \mathrm{MnVO}_{6}$ (SMVO) ground state, as the initial unrelaxed structures, taken from ref. 26. Full structural optimization calculations including atomic positions and lattice parameters were performed for the tetragonal structure with rock-salt and layered ordering of $\mathrm{B}$ and $\mathrm{B}^{\prime}$ cationic sites (Mn and $\mathrm{V}$ ) of CMVO and BMVO compounds. The DFT-calculated total energies of $\mathrm{A}_{2} \mathrm{MnVO}_{6}(\mathrm{~A}=\mathrm{Ca}$, $\mathrm{Ba})$ double perovskites showed that the ground state in both compounds is associated with the layer ordered structure (Table 1). The optimized structures with a two-formula-unit supercell of the double perovskites CMVO and BMVO (20 atoms) are shown in Fig. 2. The six oxygen atoms surrounding $\mathrm{Mn}$ and $\mathrm{V}$ sites provide an octahedral environment and the $\mathrm{MnO}_{6}$ and $\mathrm{VO}_{6}$ octahedra layers alternate along the $c$ axes.

Table 1 summarizes the optimized crystal structure parameters of $\mathrm{A}_{2} \mathrm{MnVO}_{6}(\mathrm{~A}=\mathrm{Ca}, \mathrm{Ba})$ double perovskites. Through the structural optimization, the initial tetragonal structure $(I 4 / m$, No. 87$)$ changes into monoclinic $(P c$, No. 7$)$ and orthorhombic (Pnma, No. 62) structures for CMVO and BMVO compounds, respectively. The layer-ordered structure has been reported for some double perovskites such as $\mathrm{Sr}_{2} \mathrm{MnVO}_{6},{ }^{26}$ $\mathrm{Ln}_{2} \mathrm{CuSnO}_{6}$ ( $\mathrm{Ln}=\mathrm{La}, \mathrm{Pr}$, and $\left.\mathrm{Nd}\right),{ }^{28} \mathrm{Ca}_{2} \mathrm{FeMnO}_{6},{ }^{29}$ and $\mathrm{Y}_{2} \mathrm{CrMnO}_{6},{ }^{30}$ in previous experimental and theoretical studies.

Since CMVO and BMVO compounds have not been experimentally prepared yet, the formation energies $\left(E_{\mathrm{f}}\right)$, which are 
Table 1 The optimized cell parameters, bond length averages, bond angles, distortion parameters, relative total energies, and formation energies of $\mathrm{A}_{2} \mathrm{MnVO}_{6}(\mathrm{~A}=\mathrm{Ca}, \mathrm{Ba})$ double perovskites using GGA approximation, in comparison with the results of SMVO

\begin{tabular}{llll}
\hline Parameter/compound & $\mathrm{Ca}_{2} \mathrm{MnVO}_{6}$ & $\mathrm{Ba}_{2} \mathrm{MnVO}_{6}$ & $\mathrm{Sr}_{2} \mathrm{MnVO}_{6}{ }^{a}$ \\
\hline Crystal symmetry & Monoclinic & Orthorhombic & Tetragonal \\
Space group & $P c$ & Pnma & $I 4 / m$ \\
$\mathrm{t}$ & 0.97 & 1.06 & 1.00 \\
$a(\AA)$ & 5.47 & 5.80 & 5.64 \\
$b(\AA)$ & 5.54 & 5.88 & 5.64 \\
$c(\AA)$ & 7.73 & 8.33 & 7.87 \\
$\alpha\left({ }^{\circ}\right)$ & 90.21 & 90.00 & 90.00 \\
$\beta\left({ }^{\circ}\right)$ & 90.00 & 90.00 & 90.00 \\
$\gamma\left({ }^{\circ}\right)$ & 90.00 & 90.00 & 90.00 \\
$\mathrm{~V}\left(\AA^{3}\right)$ & 234.76 & 284.34 & 250.84 \\
$\langle\mathrm{~V}-\mathrm{O}\rangle(\AA)$ & 1.95 & 1.99 & 1.95 \\
$\langle\mathrm{Mn}-\mathrm{O}\rangle(\AA)$ & 2.03 & 2.16 & 2.05 \\
$\mathrm{Mn}-\mathrm{O}-\mathrm{V}\left({ }^{\circ}\right)$ & 153.15 & 165.25 & 163.60 \\
$\left.\Delta d(\mathrm{VO})_{6}\right) \times 10^{-3}$ & 11.78 & 19.62 & 12.67 \\
$\Delta d(\mathrm{MnO}) \times 10^{-3}$ & 1.62 & 4.55 & 1.29 \\
$\Delta E_{\mathrm{Layered}-\text { Rocksalt }}(\mathrm{eV})$ & -1.29 & -1.02 & -0.39 \\
$E_{\mathrm{f}}(\mathrm{eV})$ & -8.48 & -5.08 & -8.37 \\
${ }^{a} \mathrm{Ref.} 26$. & & & \\
& & &
\end{tabular}

defined as the total energy difference between the compound and its four existing elements, are computed to verify the thermodynamic stability of these double perovskites. $E_{\mathrm{f}}$ is given as: ${ }^{31}$

$$
E_{\mathrm{f}}=E_{\text {tot }}\left(\mathrm{A}_{2} \mathrm{MnVO}_{6}\right)-\left(2 E_{\mathrm{A}}+E_{\mathrm{Mn}}+E_{\mathrm{V}}+6 E_{\mathrm{O}}\right)
$$

here $E_{\text {tot }}\left(\mathrm{A}_{2} \mathrm{MnVO}_{6}\right)$ is the total energy of the CMVO and BMVO compounds and $E_{\mathrm{A}}, E_{\mathrm{Mn}}, E_{\mathrm{V}}$ and $E_{\mathrm{O}}$ are the total energies per atom of elements in their pure bulk form. The negative values of the $E_{\mathrm{f}}$ listed in Table 1 reveal the thermodynamic stability of the compounds which means that they could be synthesized more suitably.

The tolerance factor $t$ can approximately predict the crystal structures of these new compounds by using the ionic radius of $\mathrm{Ca}^{2+}(1.48 \AA), \mathrm{Ba}^{2+}(1.75 \AA), \mathrm{Mn}^{3+}(0.78 \AA), \mathrm{V}^{5+}(0.68 \AA)$, and $\mathrm{O}^{2-}(1.26 \AA)$ tabulated by Shannon, ${ }^{32}$ given by:

$$
t=\frac{\sqrt{2}\left(r_{\mathrm{A}}+r_{\mathrm{O}}\right)}{r_{\mathrm{B}}+r_{\mathrm{B}^{\prime}}+2 r_{\mathrm{O}}}
$$

where $r_{\mathrm{A}}, r_{\mathrm{B}}, r_{\mathrm{B}^{\prime}}$ and $r_{\mathrm{O}}$ are the ionic radii of each ion in $\mathrm{A}_{2} \mathrm{BB}^{\prime} \mathrm{O}_{6}$ double perovskite. ${ }^{26}$

Table 1 illustrates that the calculated tolerance factor and structural symmetry increase with increasing the A cation size $\left(r_{\mathrm{Ca}^{2+}}<r_{\mathrm{Ba}^{2+}}\right)$. Due to the mismatch between the $\mathrm{A}-\mathrm{O}$ and $\mathrm{B}\left(\mathrm{B}^{\prime}\right)-\mathrm{O}$ interatomic distances, the $\mathrm{B}\left(\mathrm{B}^{\prime}\right) \mathrm{O}_{6}$ octahedra undergo a tilting to obtain a more favorable configuration from the energetic point of view. While the A cation size gets smaller, more tilting of the $\mathrm{B}\left(\mathrm{B}^{\prime}\right) \mathrm{O}_{6}$ octahedra takes place to fill up the empty spaces around the A cations, resulting in more deviation of the $\mathrm{B}-\mathrm{O}-\mathrm{B}^{\prime}$ bond angle from $180^{\circ}$. An increase in the tilting is observed by decreasing the tolerance factor as the $\mathrm{Mn}-\mathrm{O}-\mathrm{V}$ bond angles are $153.15^{\circ}$ and $165.25^{\circ}$ in CMVO and BMVO, respectively (see Table 1 and Fig. 2). The $\mathrm{Mn}-\mathrm{O}-\mathrm{V}$ bond angle values are comparable with the values reported for ruthenate oxide $\left(\mathrm{SrCaRu}_{2} \mathrm{O}_{6}\right)$ polar metal. ${ }^{11}$ Moreover, the lattice constants and cell volume increase with an increasing size of A site cations. A strong distortion in the $\mathrm{BO}_{6}$ octahedra compared to the ideal cubic structure is observed. One can see from Fig. 2 that the $\mathrm{MnO}_{6}$ octahedra are significantly larger than the $\mathrm{VO}_{6}$ octahedra. It is well known that as a consequence of the octahedra crystal field, the five-fold degenerate $3 \mathrm{~d}$ orbitals split into nondegenerate higher energy $\mathrm{e}_{\mathrm{g}}\left(\mathrm{d}_{x^{2}-y^{2}}\right.$ and $\left.\mathrm{d}_{z^{2}}\right)$ and lower energy $\mathrm{t}_{2 g}\left(\mathrm{~d}_{x y}, \mathrm{~d}_{x z}\right.$, and $\left.\mathrm{d}_{y z}\right)$ states. For both compounds, the odd number of electrons in the $e_{g}$ orbital causes an elongation of the $\mathrm{MnO}_{6}$ octahedra along the $c$ axis as a result of the JT character of $\mathrm{Mn}^{3+}\left(\mathrm{t}_{2 \mathrm{~g}}^{3} \mathrm{e}_{\mathrm{g}}^{1}\right)$ removing the degeneracy between $\mathrm{d}_{x^{2}-y^{2}}$ and $\mathrm{d}_{z^{2}}$ states (Fig. $2 \mathrm{~b}$ ) leading to a more stable system with lower energy configuration and reduced symmetry. ${ }^{33}$ Nevertheless, the bond lengths deviate the most from the average $\mathrm{B}-\mathrm{O}$ bond length value for the $\mathrm{VO}_{6}$ octahedra compared to $\mathrm{MnO}_{6}$ in both CMVO and BMVO compounds. The distortion parameters $(\Delta d)$, concerning the deviation of B-O distance with respect to the average $\langle\mathrm{B}-\mathrm{O}\rangle$ value, are calculated for $\mathrm{MnO}_{6}$ and $\mathrm{VO}_{6}$ octahedra as ${ }^{27,34}$

$$
\Delta d=\frac{1}{6} \sum_{n=1}^{6}\left[\frac{d_{n}-\langle d\rangle}{\langle d\rangle}\right]^{2},
$$

where $d_{n}$ is $\mathrm{B}-\mathrm{O}$ distance and $\langle d\rangle$ are the average $\mathrm{B}-\mathrm{O}$ bond lengths. The calculated $\Delta d$, listed in Table 1, indicates that more distortion in the $\mathrm{BO}_{6}$ octahedra takes place for $\mathrm{BMVO}$ compared to CMVO, which is the result of the chemical pressure due to the oversized A-site cations $(t>1)$. The obtained values for distortion parameters are a clear sign of the JT distortion in CMVO and BMVO which are as the order of reported values in the systems including $\mathrm{Mn}^{3+}\left(\mathrm{t}_{2 \mathrm{~g}}^{3} \mathrm{e}_{\mathrm{g}}^{1}\right)$ and $\mathrm{Cu}^{2+}\left(\mathrm{t}_{2 \mathrm{~g}}^{6} \mathrm{e}_{\mathrm{g}}^{3}\right) \mathrm{JT}_{\mathrm{T}}$ ions. ${ }^{34}$

Furthermore, the magnitude of $\mathrm{VO}_{6}$ octahedra distortion parameter is more than $\mathrm{MnO}_{6}$ for both compositions. One can attribute this to the Second-order Jahn-Teller effect (SOJT) originated from the $\mathrm{d}^{0}$ electron configuration of the $\mathrm{V}^{5+}\left(3 \mathrm{~d}^{0}\right)$ ion facilitating a polar displacement of these atoms with respect to neighbouring $\mathrm{O}$ atoms in their respective octahedra. ${ }^{35}$ Such an electronic instability towards polar distortion occurs in the well-known proper FEs $\mathrm{BaTiO}_{3}, \mathrm{PbTiO}_{3}$ and $\mathrm{Pb}(\mathrm{ZrTi}) \mathrm{O}_{3}$ containing transition metal ions with empty $\mathrm{d}$ shells which can hybridize with occupied O-2p states. ${ }^{35-37}$ This octahedra tilting and distortion strongly affects the electronic interactions along $\mathrm{B}-\mathrm{O}-\mathrm{B}^{\prime}-\mathrm{O}-\mathrm{B}$ paths and therefore magnetic and electronic properties.

\subsection{Magnetic and electronic structures}

To find the most stable magnetic structure, we assign different magnetic orderings $\mathrm{FM}$, antiferromagnetic (AFM) and ferrimagnetic (FiM) for the two transition metals $\mathrm{Mn}$ and $\mathrm{V}$ as the initial magnetic states (Fig. 3). Due to the presence of 3d transition metals $\mathrm{Mn}$ and $\mathrm{V}$ with strongly localized states, we examine the correlation effects by considering Hubbard correction to describe the magnetic and electronic properties. After the full optimization in the GGA and GGA $+U$ schemes (for all considered $U$ values), the FiM and AFM1 states converge to FM and AFM2 states, respectively for both CMVO and BMVO compounds. The calculated total 
(a)

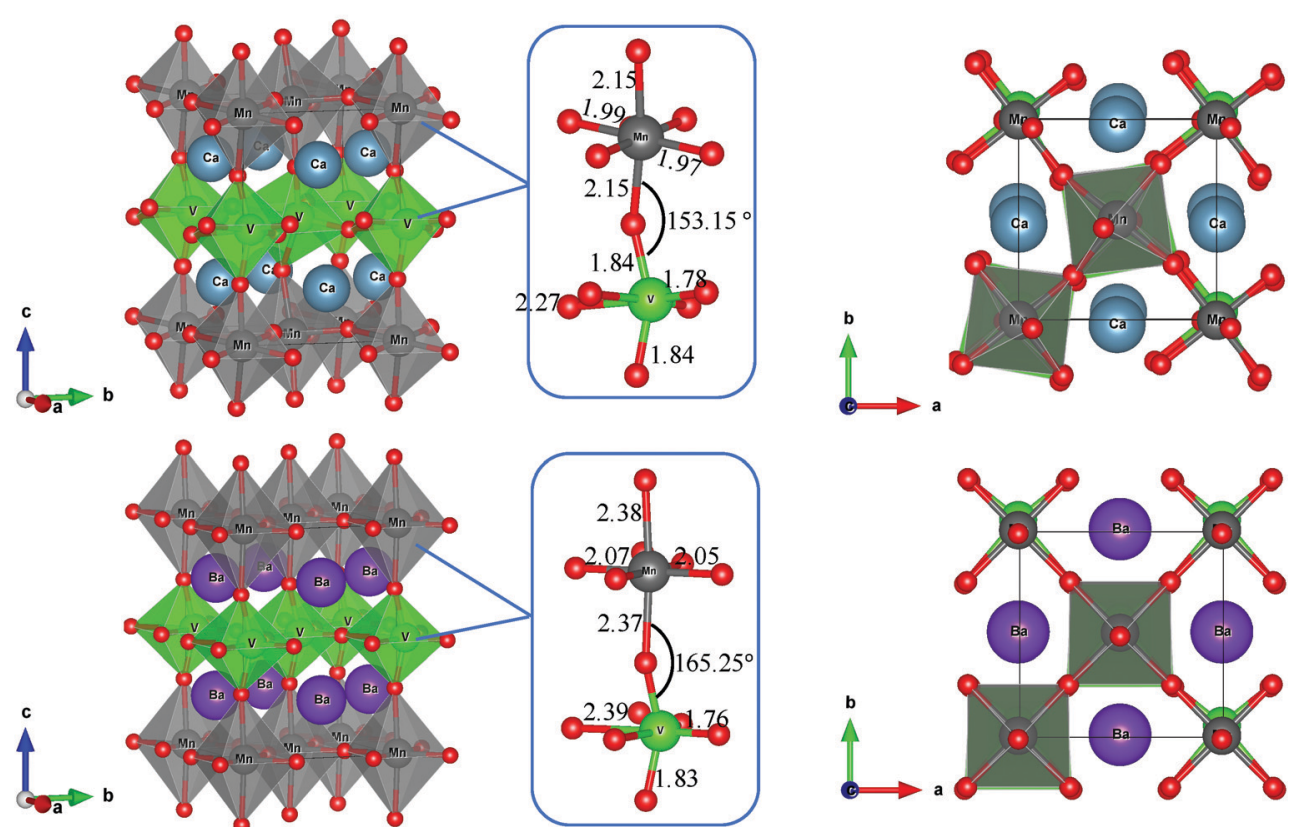

(b)

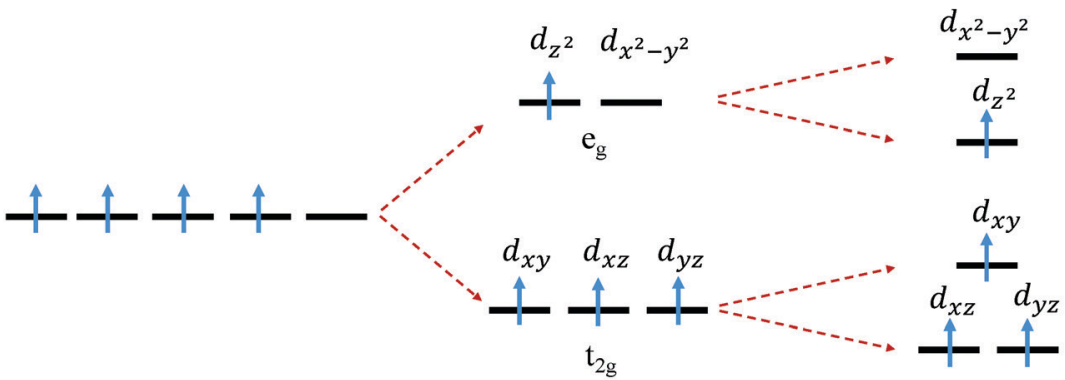

Free ion

Octahedral crystal field

Jahn-Teller elongation

Fig. 2 (a) Optimized layer ordered structures of the double perovskite $\mathrm{A}_{2} \mathrm{MnVO}_{6}(\mathrm{~A}=\mathrm{Ca}, \mathrm{Ba})$ compounds with their respective $\mathrm{MnO}_{6}$ and $\mathrm{VO}_{6}$ octahedra

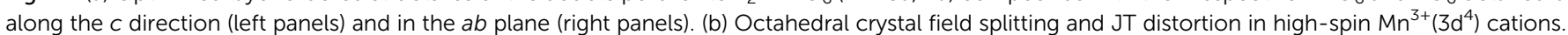
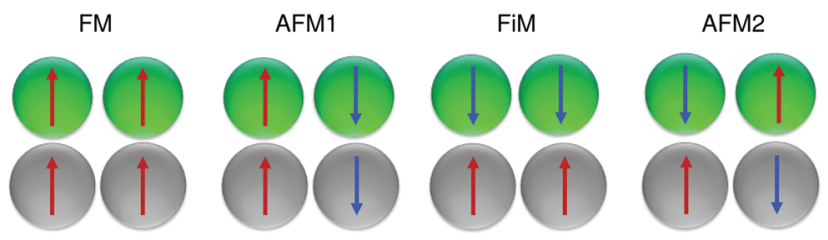

Fig. 3 The schematic diagram of different magnetic states.

energy differences $\left(\Delta E=E_{\mathrm{FM}}-E_{\mathrm{AFM}}\right)$, summarized in Table 2, show that the FM configuration is energetically favored over the AFM state for both compounds.

The magnetic ordering of CMVO and BMVO can be explained by the super-exchange interaction $\mathrm{Mn}^{3+}(3 \mathrm{~d})-\mathrm{O}^{2-}(2 \mathrm{p})$ $-\mathrm{V}^{5+}(3 \mathrm{~d})$. According to the Goodenough-Kanamori rules, ${ }^{38,39}$ the FM coupling originates from the interaction of half-filled $t_{2 g}$ and nearly empty $\mathrm{e}_{\mathrm{g}}$ in $\mathrm{Mn}^{3+}\left(3 \mathrm{~d}^{4}: \mathrm{t}_{2 \mathrm{~g}}^{3} \uparrow \mathrm{e}_{\mathrm{g}}^{1} \uparrow\right)$ with the empty $\mathrm{t}_{2 \mathrm{~g}}$ and $e_{g}$ in $\mathrm{V}^{5+}\left(3 \mathrm{~d}^{0}: \mathrm{t}_{2 \mathrm{~g}}^{0} \uparrow \mathrm{e}_{\mathrm{g}}^{0} \uparrow\right)$ through the O-2p.
Moreover, the ordering of the $\mathrm{e}_{\mathrm{g}}$ orbitals within the $a b$ planes containing JT active ions $\mathrm{Mn}^{3+}$, results in the superexchange interactions between the occupied and empty $e_{g}$ orbitals of neighboring $\mathrm{Mn}^{3+}$ ions and the electron hopping occurs between them via the $\mathrm{O}-2 \mathrm{p}$ states. This super-exchange interaction favors FM coupling between $\mathrm{Mn}$ atoms in both CMVO and BMVO systems.

The calculated total magnetic moments for CMVO and BMVO with the dominant contribution of $\mathrm{Mn}$ ions and a small contribution of $\mathrm{V}$ ions are $4.00 \mu_{\mathrm{B}}$ f.u. $^{-1}$ in both GGA and GGA $+U$ approximations. The spin magnetic moments of $0.29 \mu_{\mathrm{B}}$ and $0.10 \mu_{\mathrm{B}}$ for $\mathrm{V}$ atoms in CMVO and BMVO, respectively, result from the hybridization between $\mathrm{V}-3 \mathrm{~d}$ and $\mathrm{O}-2 \mathrm{p}$ orbitals. The valence states of $\mathrm{Mn}^{3+}(S=2)$ and $\mathrm{V}^{5+}(S=0)$ are consistent with the values of local magnetic moments. To investigate the valence configuration of CMVO and BMVO double perovskites, we use the nominal valence states of $\mathrm{A}_{2}{ }^{2+} \mathrm{Mn}^{n} \mathrm{~V}^{m} \mathrm{O}_{6}{ }^{2-}(n+m=8)$. According to the magnitude and direction of the $\mathrm{Mn} / \mathrm{V}$ spin 
Table 2 The calculated total energy difference $(\Delta E)$, total and local magnetic moments and energy gaps $\left(E_{\mathrm{g}}\right)$ for $\mathrm{A}_{2} \mathrm{MnVO} 6(\mathrm{~A}=\mathrm{Ca}$ and $\mathrm{Ba})$

\begin{tabular}{|c|c|c|c|c|c|c|c|c|c|c|}
\hline \multirow[b]{3}{*}{$U(\mathrm{Mn}, \mathrm{V})$} & \multicolumn{5}{|c|}{$\mathrm{Ca}_{2} \mathrm{MnVO}_{6}$} & \multicolumn{5}{|c|}{$\mathrm{Ba}_{2} \mathrm{MnVO}_{6}$} \\
\hline & \multirow{2}{*}{$\frac{\text { GGA }}{(0,0)}$} & \multicolumn{4}{|c|}{$\mathrm{GGA}+U$} & \multicolumn{2}{|l|}{ GGA } & \multicolumn{2}{|c|}{$\mathrm{GGA}+U$} & \multirow[b]{2}{*}{$(5,4)$} \\
\hline & & $(4,3)$ & $(4,4)$ & $(5,3)$ & $(5,4)$ & $(0,0)$ & $(4,3)$ & $(4,4)$ & $(5,3)$ & \\
\hline$\Delta E_{\mathrm{FM}-\mathrm{AFM}}(\mathrm{eV})$ & -0.20 & -0.95 & -0.23 & -0.24 & -0.25 & -0.32 & -0.49 & -0.49 & -0.54 & -0.54 \\
\hline$m_{\text {tot }}\left(\mu_{\mathrm{B}}\right.$ f.u. $\left.^{-1}\right)$ & 4.00 & 4.00 & 4.00 & 4.00 & 4.00 & 4.00 & 4.00 & 4.00 & 4.00 & 4.00 \\
\hline$m_{\mathrm{Ca} / \mathrm{Ba}}\left(\mu_{\mathrm{B}}\right)$ & -0.02 & -0.02 & -0.02 & -0.02 & -0.02 & -0.01 & -0.01 & -0.01 & -0.01 & -0.01 \\
\hline$m_{\mathrm{Mn}}\left(\mu_{\mathrm{B}}\right)$ & 4.16 & 4.52 & 4.51 & 4.60 & 4.59 & 4.41 & 4.73 & 4.72 & 4.79 & 4.79 \\
\hline$m_{\mathrm{V}}\left(\mu_{\mathrm{B}}\right)$ & 0.29 & 0.36 & 0.63 & 0.32 & 0.53 & 0.10 & 0.17 & 0.26 & 0.16 & 0.24 \\
\hline$m_{\mathrm{O}}\left(\mu_{\mathrm{B}}\right)$ & -0.06 & -0.10 & -0.16 & -0.09 & -0.14 & -0.03 & -0.05 & -0.08 & -0.05 & -0.07 \\
\hline$E_{\mathrm{g}}(\mathrm{eV})$ & 1.26 & 1.65 & 1.98 & 1.77 & 1.98 & 1.37 & 1.69 & 1.74 & 1.71 & 1.80 \\
\hline
\end{tabular}

magnetic moments, for the ideal ionic model, the $\mathrm{Mn}$ and $\mathrm{V}$ atoms can have the valence configurations of $\mathrm{Mn}^{3+}\left(3 \mathrm{~d}^{4}: \mathrm{t}_{2 \mathrm{~g}}^{3} \mathrm{e}_{\mathrm{g}}^{1}\right)$ and $\mathrm{V}^{5+}\left(3 \mathrm{~d}^{0}: \mathrm{t}_{2 \mathrm{~g}}^{0} \mathrm{e}_{\mathrm{g}}^{0}\right)$ causing the JT and SOJT distortions in their respective octahedra, as already explained. Moreover, the electron configuration of $\mathrm{Mn}^{3+}\left(3 \mathrm{~d}^{4}: \mathrm{t}_{2 \mathrm{~g}}^{3} \mathrm{e}_{\mathrm{g}}^{1}\right)$ confirms the high-spin state of these atoms. Hence, by considering the local magnetic moments in Table 2, the effective charge of $\mathrm{Mn}^{2.84+}\left(3 \mathrm{~d}^{4.16}\right)$ and $\mathrm{V}^{4.71+}\left(3 \mathrm{~d}^{0.29}\right)$ for the CMVO compound and $\mathrm{Mn}^{2.59+}\left(3 \mathrm{~d}^{4.41}\right)$ and $\mathrm{V}^{4.90+}\left(3 \mathrm{~d}^{0.10}\right)$ for BMVO are obtained in the GGA scheme as a result of the hybridization between $\mathrm{Mn}(\mathrm{V})-3 \mathrm{~d}$ and $\mathrm{O}-2 \mathrm{p}$ orbitals. Also, the effective charges of $\mathrm{O}^{1.91-}$ and $\mathrm{O}^{1.90-}$ are predicted for CMVO and BMVO compounds, respectively. The sign of the spin magnetic moments listed in Table 2, implies that both Mn and $\mathrm{V}$ possess the same alignment and thus form FM configurations. Also, the integer value of the total magnetic moment indicates that at least one of the spin channels has a band gap in the electronic DOS, which is the characteristic of HM materials. As expected, the local magnetic moments increase by applying the Hubbard approximation, while the total magnetic moments still remain as a robust integer value for the different $U$ parameters (Table 2). The predicted magnetic and electronic features for CMVO and $\mathrm{BMVO}$ here quite resemble that for $\mathrm{Ba}_{2} \mathrm{MnTaO}_{6},{ }^{40} \mathrm{~Pb}_{2} \mathrm{FeReO}_{6},{ }^{41}$ and $\mathrm{A}_{2} \mathrm{MnB}^{\prime} \mathrm{O}_{6}(\mathrm{~A}=\mathrm{Ca}$, Sr; $\left.\mathrm{B}^{\prime}=\mathrm{Sb}, \mathrm{Ta}\right),{ }^{42,43}$ implying the presence of HM ferromagnetism as a generic feature for a broad class of the ordered double perovskite compounds.

To indicate the nature of CMVO and BMVO compounds, the electronic structures for the majority- and minority-spin states were calculated. Fig. 4 and 5 illustrate the spin-polarized total and partial DOS of $\mathrm{A}_{2} \mathrm{MnVO}_{6}(\mathrm{~A}=\mathrm{Ca}, \mathrm{Ba})$ double perovskites calculated using GGA and GGA $+U$ methods, respectively. The positive and negative values of the DOS represent spin up and down channels, respectively. As can be seen from Fig. 4, the ground states of the CMVO and BMVO double perovskites have an HM nature; the spin up DOS crosses the Fermi level, while the spin-down channel forms an energy gap of about $1.26 \mathrm{eV}$ and $1.37 \mathrm{eV}$ for CMVO and BMVO, respectively resulting in full $(100 \%)$ spin-polarization of the conduction electrons at the Fermi level. The HM nature has been reported in several alkaline-earth metal-based double perovskites. ${ }^{8,26,44}$ For both compounds, at the energy range of -2 to $0 \mathrm{eV}$ in the spin-up channel, the main contribution originates from $\mathrm{O}-2 \mathrm{p}$ states hybridized with dominant $\mathrm{Mn}-3 \mathrm{~d}$ electrons and a much smaller number of the V-3d and Ca-3p/Ba-5p electrons. In the GGA scheme, the weak magnetic moment of the $\mathrm{V}$ atom is caused by the hybridization between $\mathrm{V}-\mathrm{e}_{\mathrm{g}}$ and $\mathrm{O}-2 \mathrm{p}$ states, inducing a finite DOS at the Fermi level, as shown in Fig. 4.

The Mn-3d and O-2p hybridization is responsible for the HM behaviour observed in CMVO and BMVO. The hybridization of $\mathrm{V}$ and $\mathrm{Mn}$ 3d states indicates covalent $\mathrm{V}-\mathrm{O}$ and $\mathrm{Mn}-\mathrm{O}$ bonds in the structures. The V-3d states are nearly empty in this energy region, which indicates the $\mathrm{V}^{5+}\left(3 \mathrm{~d}^{0}\right)$ valence state. Above the Fermi level, the unoccupied V-3d states lie closer to the Fermi level in comparison with the unoccupied $\mathrm{Mn}-3 \mathrm{~d}$ states in both compounds, which is similar to the $\mathrm{Mn}$ and $\mathrm{V}$ behaviour in SMVO double perovskite. ${ }^{26}$ In BMVO, the Fermi level is closer to the valence band maximum compared to the CMVO.

Including the electron correlation through GGA+U (Fig. 5) indicates an HM behaviour for CMVO and BMVO with an enhanced bandgap in the spin-down channel for all $U$ values (see Table 2). By applying the GGA $U$ scheme for both compounds, the PDOS of Mn-3d and V-3d pushed away from the Fermi level at both spin channels. One can see that the higher $U$ parameter causes more shift to occur in the $3 \mathrm{~d}$ states of $\mathrm{Mn}(\mathrm{V})$ atoms resulting in an energy gap enhancement. Due to the dominant contribution of the O-2p orbital below the Fermi level, the divergence of $\mathrm{Mn}(\mathrm{V})-3 \mathrm{~d}$ states from the Fermi energy does not affect the bandgap opening of the spin-down channel. In contrast, for the energies above the Fermi level, the shifted $\mathrm{V}-3 \mathrm{~d}$ state plays a key role in controlling the spin-down channel's band gap value.

\subsection{Charge density analysis}

The electronic charge density was calculated using GGA approximation to visualize the nature of the bond character and explain the charge transfer of CMVO and BMVO double perovskites.

The charge density difference, which is defined as the difference between charge distribution of relaxed and initial structures, is represented for the (001) planes containing $\mathrm{Mn}$ and $\mathrm{V}$ ions in Fig. 6. These plots describe the loss or gain of the charge on a specific atom as in the corresponding color scales, and white color $(+0.01)$ corresponds to the maximum charge accumulating site. ${ }^{26}$ The charge density plots show the maximum density near the oxygen atoms because of their large electronegative nature. For both CMVO and BMVO, the charge sharing between the $\mathrm{Mn}-\mathrm{O}$ and $\mathrm{V}-\mathrm{O}$ atoms in $\mathrm{MnO}_{6}$ and $\mathrm{VO}_{6}$ octahedra confirms that the bonding is covalent, which is 
(a)

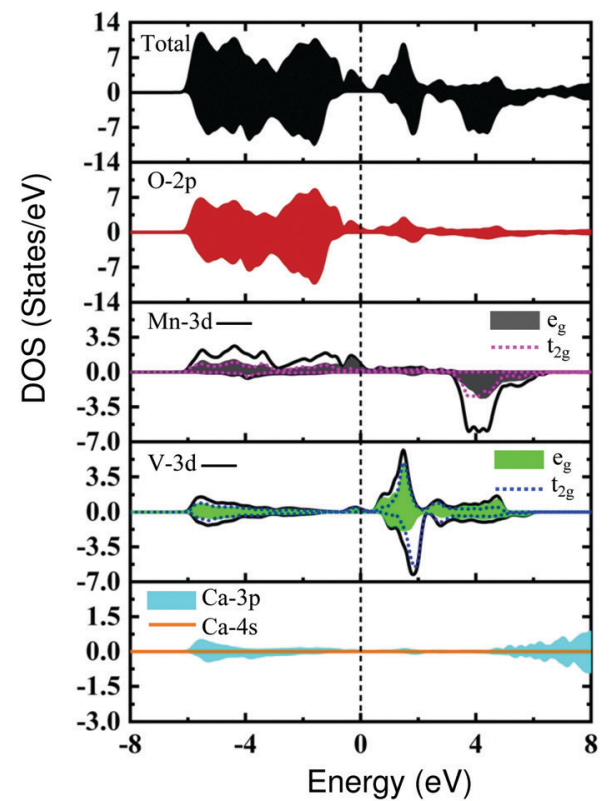

(b)

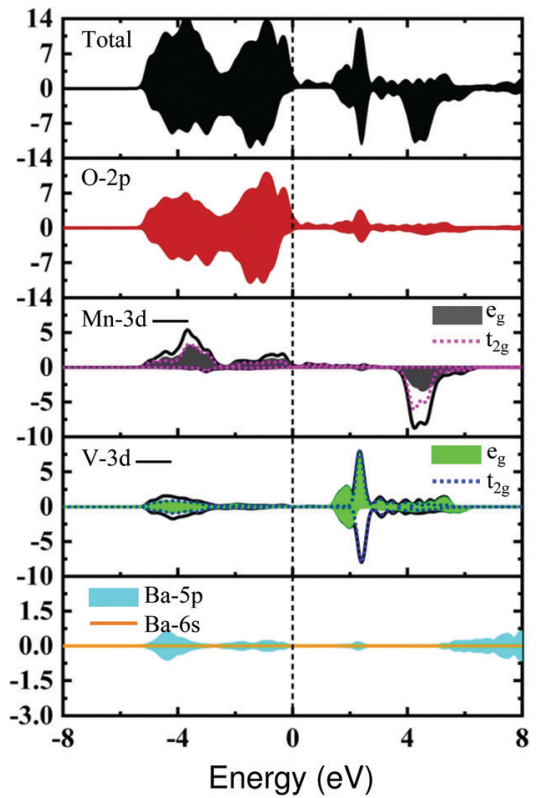

Fig. 4 Calculated total and partial density of states for (a) CMVO and (b) BMVO using the GGA approach. The vertical dashed line denotes the Fermi level which is set to zero energy.

(a)

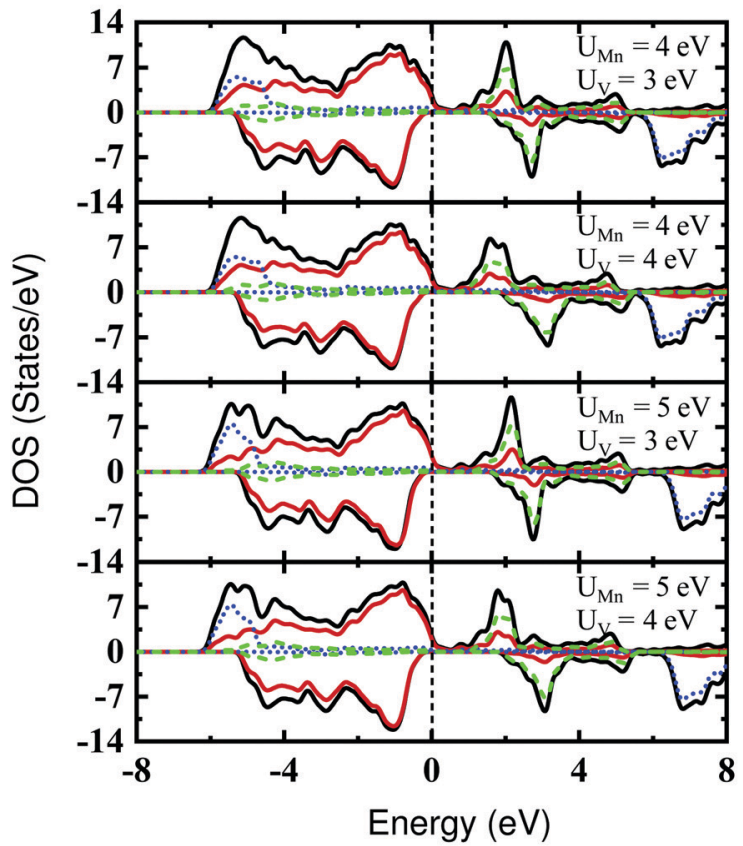

Total, (b)

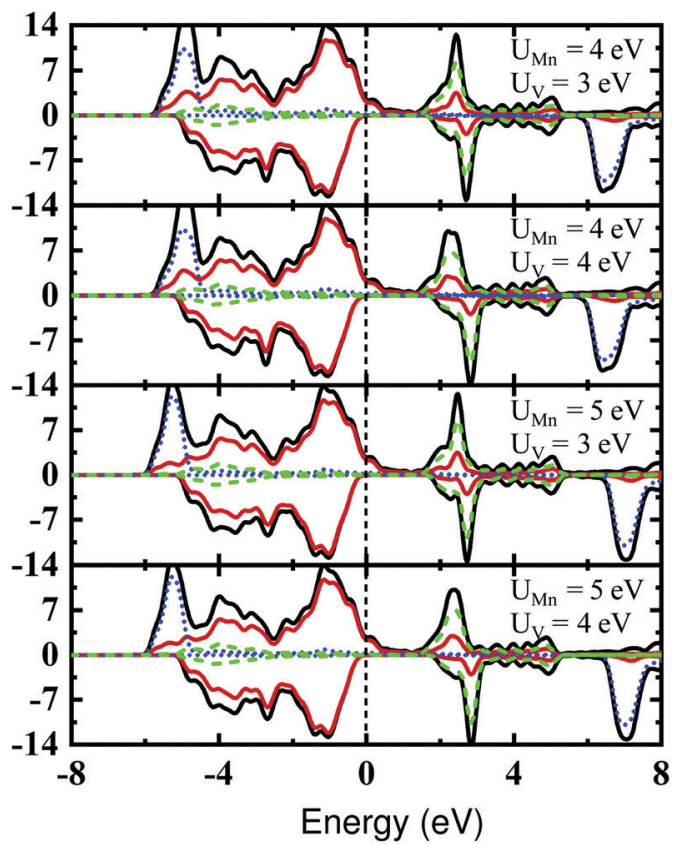

$\mathrm{Mn}-3 \mathrm{~d}$,

. V-3d

Fig. 5 Calculated total and partial density of states for (a) CMVO and (b) BMVO using the GGA+U approach. The vertical dashed line denotes the Fermi level which is set to zero energy.

essentially due to the hybridization of $\mathrm{Mn} / \mathrm{V}-3 \mathrm{~d}$ and $\mathrm{O}-2 \mathrm{p}$ states. From the symmetric charge distribution around A-site cations in Fig. 6(a), we can figure out that $\mathrm{Ca}$, and $\mathrm{Ba}$ are isolated from the other atoms, substantiating the ionic bonding nature with 
(a)

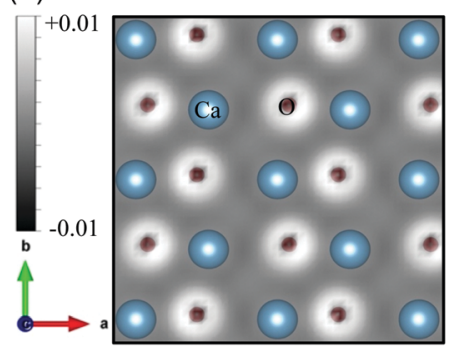

(b)

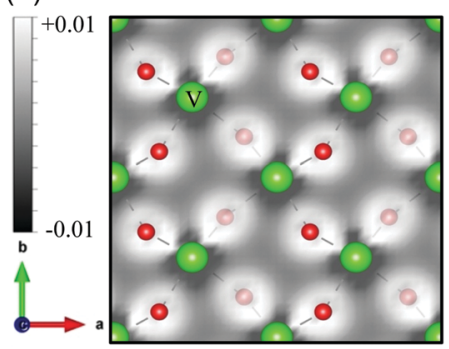

(c)

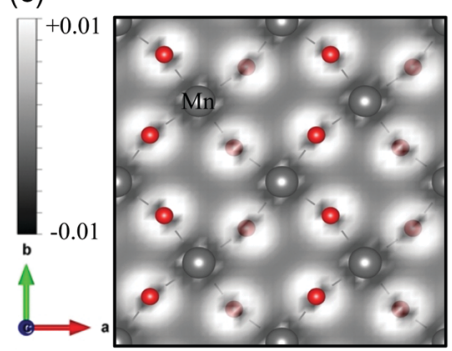

Fig. 6 The electron charge density difference contours of the CMVO (left panels) and BMVO (right panels) in the (001) plane including (a) $\mathrm{Ca} / \mathrm{Ba}$, (b) $\mathrm{V}$ and (c) Mn ions.

oxygen atoms. The electronegativity decreases from $\mathrm{Ca}$ to $\mathrm{Ba}$, resulting in a more localized and symmetric charge distribution around the $\mathrm{O}$ atom connecting to the $\mathrm{Ba}$ atom (compared to $\mathrm{Ca}$ ), which shows a slightly higher tendency for the $\mathrm{Ba}-\mathrm{O}$ bond to be ionic. The non-centrosymmetric displacement of $\mathrm{V}$ atoms in their respective octahedra in CMVO and BMVO compounds can be observed in Fig. 6(b), indicating the potential electric polarization in these materials as was previously seen in the socalled polar metals. ${ }^{12,13}$ Furthermore, Fig. 6(b and c) represent a larger magnitude of tilting for $\mathrm{MnO}_{6}$ and $\mathrm{VO}_{6}$ octahedra in CMVO compared to that of BMVO.

\section{Conclusions}

In summary, we performed first-principles calculations implemented in the SIESTA code within the GGA and GGA $+U$ schemes to propose new double perovskites $\mathrm{A}_{2} \mathrm{MnVO}_{6}(\mathrm{~A}=\mathrm{Ca}$ and $\mathrm{Ba}$ ) with the coexistence of half-metallicity and polar distortions. The total energy calculations revealed that the layer ordered structure is energetically favoured over the rock-salt one for both CMVO and BMVO compounds. The optimized crystal structures of CMVO and BMVO turned out to be monoclinic and orthorhombic. For both compositions, the JT effect in $\mathrm{MnO}_{6}$ octahedra and non-centrosymmetric displacement of $\mathrm{V}$ atoms in the $\mathrm{VO}_{6}$ octahedra (SOJT effect) were observed as a result of the electron configurations of $\mathrm{Mn}^{3+}\left(3 \mathrm{~d}^{4}: \mathrm{t}_{2 \mathrm{~g}}^{3} \mathrm{e}_{\mathrm{g}}^{1}\right)$ and $\mathrm{V}^{5+}\left(3 \mathrm{~d}^{0}\right)$, respectively. From the spin-polarized calculations, the FM ground state with a total magnetic moment of $4.00 \mu_{\mathrm{B}}$ f.u. ${ }^{-1}$ was obtained for CMVO and BMVO. The DOS calculations indicated the HM nature with complete (100\%) spin polarization. To examine the robustness of our prediction that CMVO and BMVO are polar FM half-metals, we applied the Hubbard correction on Mn and V 3d orbitals, and the results showed that the polar distortions are weakly affected by the correlation effects. Therefore, our investigations predicted the concurrence of half-metallicity and polar nature in CMVO and BMVO double perovskites, which is a rare behaviour, making them suitable candidates for future spintronic device applications. We hope that this work will provide a material platform and initiate further experimental efforts to synthesize new FM polar halfmetal double perovskites.

\section{Conflicts of interest}

There are no conflicts to declare.

\section{Acknowledgements}

The computational calculations contributing to this work were carried out with the support of the UCloud Services provided by the eSience Center at SDU.

\section{Notes and references}

1 F. Sher, A. Venimadhav, M. G. Blamire, K. Kamenev and J. P. Attfield, Chem. Mater., 2005, 17, 176-180.

2 S. Vasala and M. Karppinen, Prog. Solid State Chem., 2015, 43, 1-36.

3 J.-H. Park, E. Vescovo, H.-J. Kim, C. Kwon, R. Ramesh and T. Venkatesan, Nature, 1998, 392, 794-796.

4 G. Vaitheeswaran, V. Kanchana and A. Delin, Appl. Phys. Lett., 2005, 86, 032513.

5 Y. Shimakawa, M. Azuma and N. Ichikawa, Materials, 2011, 4, 153-168.

6 R. Mukherjee, B. Ghosh, S. Saha, C. Bharti and T. Sinha, J. Rare Earths, 2014, 32, 334-342.

7 A. Hirohata, K. Yamada, Y. Nakatani, I.-L. Prejbeanu, B. Diény, P. Pirro and B. Hillebrands, J. Magn. Magn. Mater., 2020, 509, 166711.

8 Z. Szotek, W. M. Temmerman, A. Svane, L. Petit and H. Winter, Phys. Rev. B: Condens. Matter Mater. Phys., 2003, 68, 104411.

9 R. A. de Groot, F. M. Mueller, P. G. van Engen and K. H. J. Buschow, Phys. Rev. Lett., 1983, 50, 2024-2027.

10 H. Chen and A. Millis, Sci. Rep., 2017, 7, 6142.

11 D. Puggioni and J. M. Rondinelli, Nat. Commun., 2014, 5, 3432.

12 W. X. Zhou and A. Ariando, Jpn. J. Appl. Phys., 2020, 59, SI0802. 
13 Y.-W. Fang and H. Chen, Commun. Mater., 2020, $1,1$.

14 C. Zhang, L. Zhang, C. Tang, S. Sanvito, B. Zhou, Z. Jiang and A. Du, Phys. Rev. B, 2020, 102, 134416.

15 P. C. Rout and V. Srinivasan, Phys. Rev. Lett., 2019, 123, 107201.

16 D. Puggioni, A. Stroppa and J. M. Rondinelli, Phys. Rev. Mater., 2018, 2, 114403.

17 J. M. Soler, E. Artacho, J. D. Gale, A. García, J. Junquera, P. Ordejón and D. Sánchez-Portal, J. Phys.: Condens. Matter, 2002, 14, 2745-2779.

18 D. Sánchez-Portal, P. Ordejón, E. Artacho and J. M. Soler, Int. J. Quantum Chem., 1997, 65, 453-461.

19 P. Ordejón, E. Artacho and J. M. Soler, Phys. Rev. B: Condens. Matter Mater. Phys., 1996, 53, R10441-R10444.

20 J. P. Perdew, K. Burke and M. Ernzerhof, Phys. Rev. Lett., 1996, 77, 3865-3868.

21 H. J. Monkhorst and J. D. Pack, Phys. Rev. B: Solid State, 1976, 13, 5188-5192.

22 J. P. Perdew, R. G. Parr, M. Levy and J. L. Balduz, Phys. Rev. Lett., 1982, 49, 1691-1694.

23 A. I. Liechtenstein, V. I. Anisimov and J. Zaanen, Phys. Rev. B: Condens. Matter Mater. Phys., 1995, 52, R5467-R5470.

24 K. Yang, D. I. Khomskii and H. Wu, Phys. Rev. B, 2018, 98, 085105.

25 S. Chen, Z. Xiao, P. Lee, Y. Liu and Y. Wang, Phys. B, 2011, 406, 2783-2787.

26 N. Rahmani, M. E. Ghazi, M. Izadifard, D. Wang, A. Shabani and B. Sanyal, J. Phys.: Condens. Matter, 2019, 31, 475501.

27 N. Rahmani, M. Ghazi, M. Izadifard and B. Sanyal, J. Magn. Magn. Mater., 2021, 519, 167492.
28 M. Azuma, S. Kaimori and M. Takano, Chem. Mater., 1998, 10, 3124-3130.

29 Y. Hosaka, N. Ichikawa, T. Saito, P. Manuel, D. Khalyavin, J. P. Attfield and Y. Shimakawa, J. Am. Chem. Soc., 2015, 137, 7468-7473.

30 W. Wang, F. Liu, X. Zhang, X. Shen, Y. Yao, Y. Wang, B. Liu, X. Liu and R. Yu, Inorg. Chem. Front., 2018, 5, 217-224.

31 A. A. Emery and C. Wolverton, Sci. Data, 2017, 4, 170153.

32 R. D. Shannon, Acta Crystallogr., Sect. A: Cryst. Phys., Diffr., Theor. Gen. Crystallogr., 1976, 32, 751-767.

33 Y. Yamashita and K. Ueda, Phys. Rev. Lett., 2000, 85, 4960-4963.

34 M. W. Lufaso and P. M. Woodward, Acta Crystallogr., Sect. B: Struct. Sci., Cryst. Eng. Mater., 2004, 60, 10-20.

35 I. B. Bersuker, Chem. Rev., 2001, 101, 1067-1114.

36 A. Filippetti and N. A. Hill, Phys. Rev. B: Condens. Matter Mater. Phys., 2002, 65, 195120.

37 J. M. Rondinelli, A. S. Eidelson and N. A. Spaldin, Phys. Rev. B: Condens. Matter Mater. Phys., 2009, 79, 205119.

38 J. B. Goodenough, J. Phys. Chem. Solids, 1958, 6, 287-297.

39 J. Kanamori, J. Phys. Chem. Solids, 1959, 10, 87-98.

40 M. M. Saad and H.-E. A. Elhag, Results Phys., 2018, 9, 793-805.

41 Y. Zhang, V. Ji and K.-W. Xu, Phys. B, 2012, 407, 2617-2621.

42 T. K. Mandal, V. V. Poltavets, M. Croft and M. Greenblatt, J. Solid State Chem., 2008, 181, 2325-2331.

43 S. A. Dar, V. Srivastava and U. K. Sakalle, J. Magn. Magn. Mater., 2019, 484, 298-306.

44 D. Rai, A. Shankar, M. Ghimire, Sandeep and R. Thapa, Comput. Mater. Sci., 2015, 101, 313-320. 